\title{
Uterine Corpus Hydropic Leiomyoma
}

National Cancer Institute

\section{Source}

National Cancer Institute. Uterine Corpus Hydropic Leiomyoma. NCI Thesaurus. Code C126975.

A variant of leiomyoma arising from the uterine corpus. It is characterized by conspicuous zonal edema. Hyalinization may also be present. 